\title{
Rendimiento de la biopsia intraoperatoria por congelación en el estudio del linfonodo centinela en cáncer de mama*
}

\author{
Drs. JAIME JANS B. ${ }^{1}$, NICOLÁS ESCUDERO M. ${ }^{4}$, FELIPE LEÓN F. ${ }^{1}$, \\ AUGUSTO LEÓN R. ${ }^{1}$, FRANCISCO DOMÍNGUEZ C. ${ }^{1}$, IGNACIO GOÑI E. ${ }^{1}$, \\ NICOLÁS DROPPELMANN M. ${ }^{1}$, CÉSAR SÁNCHEZ R. ${ }^{2}$, DAVID ODDÓ B. ${ }^{3}$, \\ PAULINA ALONSO V. ${ }^{4}$, FELIPE IBÁÑEZ M. ${ }^{4}$, MAURICIO CAMUS A. ${ }^{1}$
}

1 Departamento de Cirugía Oncológica.

2 Departamento de Hematología-Oncología.

3 Departamento de Anatomía Patológica.

4 Facultad de Medicina.

Pontificia Universidad Católica de Chile.

Santiago, Chile.

Abstract

\section{Intraoperative frozen section of the sentinel lymph nodes in breast cancer}

Introduction: The sentinel lymph node (SN) biopsy has become the standard technique for staging the axillary involvement in breast cancer. The intraoperative diagnosis of lymph node metastatic involvement can immediately decide the need for axillary dissection. The main objective of this study was to determine the accuracy of intraoperative frozen section in the investigation of metastases in the SN, in patients with breast cancer, between 1999 and 2011. Materials and Methods: A retrospective study of patients operated for invasive breast cancer, in which the SN was studied with intraoperative frozen section. Results: 503 patients biopsy was performed with intraoperative frozen section. One hundred fifty-four $(30.6 \%)$ patients had metastases in the SN, of these $103(66.9 \%)$ were detected on frozen section. No significant difference in the ability to detect metastases for tumors up to $2 \mathrm{~cm}$ and tumors more than $2 \mathrm{~cm}$ was observed $(\mathrm{p}=0.86)$. The sensitivity, specificity, positive predictive value (PPV) and negative predictive value (NPV) of frozen section for detection of metastases in the SN was $66.9 \%, 99.7 \%, 99 \%$ and $87 \%$, respectively. Conclusions: The accuracy of intraoperative biopsy for detection of metastases in the $\mathrm{SN}$ in our center is comparable to that described in the literature, being effective only for the investigation of macrometastases (MA). Given the low frequency of metastases in the SN in tumors up to $2 \mathrm{~cm}$, associated with a relatively high number of micro (MI) and submicrometastasis (SM) and false negatives, it seems that in these tumors intraoperative biopsy is unnecessary.

Key words: Breast cancer, sentinel lymph node biopsy, frozen section, intraoperative evaluation.

*Recibido el 9 de septiembre de 2012 y aceptado para publicación el 27 de octubre de 2012.

Los autores declaran no tener conflicto de interés.

Correspondencia: Dr. Mauricio Camus A.

Marcoleta 352, Santiago, Chile. Fax: (2) 639-6395

mcamus@med.puc.cl 


\section{Resumen}

Introducción: La biopsia del linfonodo centinela (LC) se ha convertido en la técnica estándar para estadificar el compromiso axilar en el cáncer de mama. El diagnóstico intraoperatorio del compromiso metastásico del linfonodo permite decidir en forma inmediata la necesidad de disección axilar. El objetivo general de este estudio fue determinar el rendimiento de la biopsia intraoperatoria por congelación en la pesquisa de metástasis en el LC, en pacientes con cáncer de mama, entre 1999 y 2011. Material y Métodos: Estudio retrospectivo de las pacientes operadas por cáncer de mama invasor, en que se realizó biopsia del LC, con estudio intraoperatorio por congelación. Resultados: 503 pacientes con biopsia del LC con estudio intraoperatorio. Ciento cincuenta y cuatro $(30,6 \%)$ pacientes tuvieron metástasis en el LC, de estos $103(66,9 \%)$ fueron detectadas en la biopsia rápida. No hubo diferencias significativas en la capacidad de detección para los tumores de hasta $2 \mathrm{~cm}$ y los tumores mayores de $2 \mathrm{~cm}(\mathrm{p}=0,86)$. La sensibilidad, especificidad, valor predictivo positivo (VPP) y valor predictivo negativo (VPN) de la biopsia intraoperatoria para la detección de metástasis en el LC fue de $66,9 \%, 99,7 \%, 99 \%$ y $87 \%$, respectivamente. Conclusiones: El rendimiento obtenido es comparable al descrito en la literatura, siendo efectiva sólo para la pesquisa de macrometástasis (MA). Dada la baja frecuencia de metástasis en el LC en tumores de hasta $2 \mathrm{~cm}$, asociado a un número relativamente alto de micro (MI) y submicrometástasis (SM) y de falsos negativos, nos parece que, en estos casos, la utilización de la biopsia intraoperatoria del LC es innecesaria.

Palabras clave: Cáncer de mama, linfonodo centinela, biopsia por congelación, evaluación intraoperatoria.

\section{Introducción}

El estudio del linfonodo centinela (LC) es en la actualidad la técnica estándar de referencia para determinar el compromiso de los linfonodos axilares, en los pacientes con cáncer de mama ${ }^{1-4}$, con morbilidad mucho menor que la disección axilar ${ }^{5-9}$. La evaluación intraoperatoria del LC con biopsia por congelación, permite determinar el compromiso metastásico durante la misma intervención quirúrgica, permitiendo continuar con la disección axilar terapéutica si el LC presenta metástasis, evitando así la necesidad de una segunda operación. El estudio de la biopsia intraoperatoria por congelación es el método más comúnmente utilizado, sin embargo, la mejor técnica de evaluación anatomopatológica no se ha estandarizado ${ }^{10,11}$. Las guías de la Sociedad Americana de Oncología Clínica (ASCO) del año 2005, para la evaluación intraoperatoria del linfonodo centinela, indican que a pesar del riesgo de destrucción tisular del linfonodo centinela con el estudio por congelación, pareciera ser el método de elección para cirujanos y patólogos con experiencia ${ }^{12}$.

Los resultados de la biopsia intraoperatoria por congelación, han demostrado ser variados, con certeza y sensibilidad globales cercanas a $90 \%$ y $68 \%$, respectivamente, siendo estas cifras mayores en los casos de macrometástasis (MA) que en las micro (MI) y submicrometástasis (SM), en las que los resultados son deficientes y poco confiables ${ }^{13}$.

El objetivo general de este estudio fue determinar el rendimiento de la biopsia intraoperatoria por congelación en la pesquisa de metástasis en el LC, realizado a pacientes con cáncer de mama invasor.
Los objetivos específicos fueron:

- Establecer el compromiso metastásico axilar según el tamaño tumoral.

- Determinar el tipo de metástasis (MA, MI o SM) según el tamaño tumoral.

- Establecer la capacidad de pesquisa de metástasis axilares según tamaño tumoral.

- Establecer la sensibilidad, especificidad, VPP y VPN de la biopsia intraoperatoria por congelación en la pesquisa de metástasis en el LC en forma global y para MA, MI y SM.

\section{Material y Método}

\section{Diseño}

Estudio de pruebas diagnósticas. Revisión retrospectiva de registro prospectivo.

\section{Pacientes}

Se incluyó a todas las pacientes operadas por cáncer de mama invasor entre mayo de 1999 y diciembre de 2011 en el Hospital Clínico de la Pontificia Universidad Católica de Chile, en que se realizó biopsia del LC, con estudio intraoperatorio por congelación del mismo. La biopsia diferida se realizó con cortes seriados con tinción de Hematoxilina y Eosina y estudio de inmunohistoquímica para pancitoqueratinas. La técnica de biopsia del LC empleada en la mayoría de las pacientes fue la técnica mixta (linfocintigrafía con inyección de Nanoscint ${ }^{\circledR}$ marcado con Tecnecio ${ }^{99 m}$, con uso de sonda intraoperatoria detectora de radiactividad y tinta azul patente al $1 \%$ ). Las pacientes en las que 
el LC no fue estudiado con biopsia intraoperatoria fueron excluidas.

\section{Fuentes}

Los datos se recolectaron de los registros de fichas clínicas y registros de biopsias del Departamento de Anatomía Patológica de la Pontificia Universidad Católica de Chile.

\section{Variables}

Se realizó una planilla Excel con los datos considerando: edad, tamaño tumoral, histología, resultados de biopsia intraoperatoria por congelación y diferida, presencia de MA, MI y SM. Se consideró MA aquellas mayores de $2 \mathrm{~mm}$, MI aquellas mayores de $0,2 \mathrm{~mm}$ y hasta $2 \mathrm{~mm}$ y $\mathrm{SM}$ aquellas de hasta $0,2 \mathrm{~mm}$.

\section{Análisis estadístico}

Para el análisis estadístico se utilizó el programa Statcalc V.6. Se realizó estadística descriptiva. Para comparar pruebas, se realizó pruebas de hipótesis paramétricas y no paramétricas según la determinación de normalidad. Se calculó la sensibilidad, especificidad, VPP y VPN y las razones de probabilidad positiva y negativa de la biopsia intraoperatoria por congelación considerando como estándar de oro el resultado de la biopsia diferida del mismo. Se consideró estadísticamente significativo un nivel de confianza del $95 \%$ con un valor $\mathrm{p}<0,05$.

\section{Resultados}

Durante el período analizado, a 717 pacientes se les realizó biopsia del LC. De éstas, en $528(73,6 \%)$ se hizo biopsia intraoperatoria. En 25 casos el estudio del LC fue realizado en pacientes con carcinoma in situ a las que se les realizó mastectomía total con o sin reconstrucción mamaria inmediata, siendo negativo en todas ellas, motivo por el cual se excluyeron de esta revisión, considerando para el análisis sólo los 503 casos de carcinoma invasor.

La mediana de edad fue de 56 años (rango 29-89 años). La histología más frecuente fue carcinoma ductal invasor en 418 pacientes $(83,1 \%)$, seguido de carcinoma lobulillar invasor en 34 pacientes $(6,8 \%)$. El resto de las histologías correspondió a carcinoma mixto $=12$ pacientes, tubular $=12$ pacientes, papilar $=11$ pacientes, mucinoso $=9$ pacientes, medular $=6$ pacientes y sarcomatoide $=1$ paciente.

Ciento cincuenta y cuatro pacientes $(30,6 \%)$, tuvieron metástasis en el LC, existiendo un aumento de la proporción progresiva en relación al tamaño tumoral (Tabla 1). De los linfonodos positivos encontrados por biopsia diferida, 103/154 (66,9\%) fueron detectadas en la biopsia intraoperatoria (Tabla 2).

Dado el bajo número de casos con tumores TmicT1a y T3-T4, agrupamos a las pacientes en aquellas con tumores de hasta $2 \mathrm{~cm}$ (T1) (335 pacientes) y tumores mayores de $2 \mathrm{~cm}$ (168 pacientes). El compromiso del LC fue de 22,1\% (74/333) y de $47,6 \%$ $(80 / 168)$, respectivamente $(p=0,000000008)$. La proporción de detección de metástasis del LC en la biopsia intraoperatoria para los tumores de hasta 2 $\mathrm{cm}$ fue de $67,6 \%$ y para los tumores mayores de 2 cm fue de $66,3 \%$, sin encontrar diferencias estadísticamente significativas $(\mathrm{p}=0,86)$.

El compromiso linfonodal estuvo dado por MA en 103 casos, por MI en 21 casos y SM en 30 casos. El porcentaje de detección de la biopsia intraoperatoria por congelación para las MA fue de 90,3\% (93/103), para las MI de 33,3\% (7/21) y para las SM de 10\% (3/30), lo que mostró una capacidad de detección de MA estadísticamente significativa $(\mathrm{p}<0,0001)$.

Tabla 1. Número de pacientes con linfonodo centinela positivo, según estadio tumoral $(\mathrm{T})$ y estratificado por tamaño de la metástasis

\begin{tabular}{|lrrrrrr|}
\hline $\begin{array}{c}\text { Tamaño } \\
\text { tumoral (T) }\end{array}$ & n & MA & $\begin{array}{c}\text { Linfonodo centinela positivo en biopsia diferida } \\
\text { MI }\end{array}$ & SM & Total & \% \\
\hline T1mic & 28 & 0 & 0 & 0 & 0 & 0,00 \\
T1a & 27 & 3 & 1 & 0 & 4 & 14,81 \\
\hline T1b & 86 & 10 & 2 & 5 & 17 & 19,77 \\
\hline T1c & 194 & 34 & 7 & 12 & 53 & 27,32 \\
\hline T2 & 151 & 46 & 11 & 12 & 69 & 45,70 \\
T3 & 15 & 8 & 0 & 1 & 9 & 60,00 \\
\hline T4 & 2 & 2 & 0 & 0 & 2 & 100,00 \\
\hline Total & 503 & 103 & 21 & 30 & 154 & 30,62 \\
\hline
\end{tabular}

$\mathrm{MA}=$ Macrometástasis, $\mathrm{MI}=$ Micrometástasis, $\mathrm{SM}=$ Submicrometástasis. 
Tabla 2. Pacientes con linfonodo centinela positivo y proporción de detección a través de biopsia intraoperatoria, distribuidos según estadio $\mathrm{T}$

\begin{tabular}{|lccc|}
\hline Estadio T & $\begin{array}{c}\text { Pacientes con LC (+) } \\
\text { en biopsia diferida }\end{array}$ & \multicolumn{2}{c|}{$\begin{array}{c}\text { Pacientes con LC } \\
(+) \text { en biopsia IO } \\
\text { n }\end{array}$} \\
\hline T1mic & 0 & 0 & 0 \\
\hline T1a & 4 & 4 & 100 \\
\hline T1b & 17 & 10 & 58,8 \\
\hline T1c & 53 & 36 & 67,9 \\
\hline T2 & 69 & 45 & 65,2 \\
\hline T3 & 9 & 6 & 66,7 \\
\hline T4 & 2 & 2 & 100 \\
\hline Total & 154 & 103 & 66,9 \\
\hline
\end{tabular}

LC $(+)=$ Linfonodo centinela positivo, $\mathrm{IO}=$ intraoperatoria.

La sensibilidad, especificidad, VPP y VPN de la biopsia intraoperatoria para la detección de metástasis en el LC fue de 66,9\%, 99,7\%, 99\% y $87 \%$, respectivamente, y la razón de probabilidad positiva (RPP) y la razón de probabilidad negativa (RPN) fueron de 233,4 y 0,33; respectivamente (Tabla 3). Al estratificar según el tamaño de las metástasis en el LC, la sensibilidad, especificidad, VPP y VPN de la biopsia intraoperatoria para la detección de MA fue de $90,3 \%, 100 \%, 98,9 \%$ y $97,6 \%$, respectivamente y para MI y SM (agrupadas) de 19,6\%, $99,8 \%, 91 \%$ y $91,2 \%$, respectivamente (Tablas 4 y 5$)$.

\section{Discusión}

El objetivo del estudio intraoperatorio del LC es la identificación de metástasis en el mismo acto quirúrgico, permitiendo la disección axilar subsecuente sin necesidad de una segunda operación. Los métodos más difundidos para el estudio intraoperatorio del LC son la biopsia por congelación y la impronta citológica. Si bien sus resultados dependen de los distintos centros, sus sensibilidades se han descrito equivalentes ${ }^{14}$. En nuestro centro realizamos el estudio intraoperatorio por congelación a la gran mayoría de las pacientes, luego de que logramos establecer el estudio del LC como un estándar en la estadificación axilar de nuestra práctica quirúrgica ${ }^{15}$. Aquellas pacientes sin biopsia intraoperatoria del LC corresponden en su mayoría a aquellas a las que se les realizó disección axilar como parte del proceso de validación de la técnica.

Encontramos un compromiso linfonodal similar
Tabla 3. Tabla de contingencia de resultados de biopsia intraoperatoria y biopsia definitiva en los casos con metástasis en el LC

\begin{tabular}{|lcrrc|}
\hline & \multicolumn{5}{c|}{ Biopsia definitiva } \\
& & $(+)$ & $(-)$ & Total \\
Biopsia & $(+)$ & 103 & 1 & 104 \\
intraoperatoria & $(-)$ & 51 & 348 & 399 \\
& Total & 154 & 349 & 503 \\
\hline
\end{tabular}

Sensibilidad $=66,9 \%$, Especificidad $=99,7 \%, \mathrm{VPP}=99 \%$ y $\mathrm{VPN}=87 \%$.

Tabla 4. Tabla de contingencia de resultados de biopsia intraoperatoria y biopsia definitiva en los casos con MA en el LC

\begin{tabular}{|lcrrc|}
\hline & \multicolumn{5}{c|}{ Biopsia definitiva } \\
& & $(+)$ & $(-)$ & Total \\
Biopsia & $(+)$ & 93 & 0 & 93 \\
intraoperatoria & $(-)$ & 10 & 400 & 410 \\
& Total & 103 & 400 & 503 \\
\hline
\end{tabular}

Sensibilidad $=90,3 \%$, Especificidad $=100 \%$, VPP $=98,9 \%$ y $\mathrm{VPN}=97,6 \%$.

Tabla 5. Tabla de contingencia de resultados de biopsia intraoperatoria y biopsia definitiva en los casos con MI y SM en el LC

\begin{tabular}{|lcccc|}
\hline & & $\begin{array}{c}\text { Biopsia definitiva } \\
(+)\end{array}$ & $(-)$ & Total \\
Biopsia & $(+)$ & 10 & 1 & 11 \\
intraoperatoria & $(-)$ & 41 & 451 & 492 \\
& Total & 51 & 452 & 503 \\
\hline
\end{tabular}

Sensibilidad $=19,6 \%$, Especificidad $=99,8 \%$, VPP $=91 \%$ y $\mathrm{VPN}=91,2 \%$.

al de otros estudios $(30,6 \%)$ y se correlacionó con el aumento del tamaño tumoral. Para tumores de hasta $2 \mathrm{~cm}$, el compromiso linfonodal fue significativamente menor que para tumores mayores de $2 \mathrm{~cm}$ $(22,1 \%$ y $47,6 \%, p=0,000000008)$, sin embargo, el porcentaje de detección de metástasis a través de la biopsia intraoperatoria fue similar, lo que difiere de otros estudios ${ }^{16-18}$. Al comparar los resultados por el tamaño de las metástasis, encontramos que la sensibilidad para las MA fue significativamente mayor que para las MI y SM (agrupadas) (90,3\% y $19,6 \%$, $\mathrm{p}<0,0001$ ) lo que ha sido reportado en forma casi constante por otros autores, resaltando el bajo rendimiento del estudio intraoperatorio para la detección de estas últimas. Cipolla y cols ${ }^{16}$, en 364 pacientes 
reportaron una sensibilidad de $74 \%$. La sensibilidad por tamaño tumoral fue de 57,1\%, 69,2\% y $86,1 \%$ respectivamente, para tumores $\mathrm{T} 1 \mathrm{a} / \mathrm{b}, \mathrm{T} 1 \mathrm{c}$ y $\mathrm{T} 2 / 3$, con correlación estadísticamente significativa ( $\mathrm{p}<0,01)$. La detección de MA y MI fue de 90,4\% y $12,5 \%$, respectivamente. Liu y cols ${ }^{13}$, analizaron retrospectivamente los resultados de la biopsia rápida por congelación en 326 pacientes. La sensibilidad global fue de $60,6 \%$ y la especificidad de $100 \%$. La sensibilidad fue significativamente más baja en el grupo de MI y SM en comparación al grupo de MA $(28,9 \%$ vs $80,3 \% \mathrm{p}<0,0001)$. Además realizaron un metaanálisis con 47 estudios incluido el suyo. La sensibilidad global fue de $73 \%$ (94\% para MA y $40 \%$ para MI y SM) y la especificidad de $100 \%$. Con respecto a la especificidad, en nuestro estudio, no fue $100 \%$ debido a un caso falso positivo en que la biopsia intraoperatoria por congelación informó metástasis de carcinoma, que no fue corroborado en la biopsia diferida, lo que puede deberse a pérdida del material de biopsia en la toma de muestra por congelación.

El impacto clínico terapéutico de los falsos negativos en la biopsia intraoperatoria parece no ser tan relevante, según algunos reportes. Geertsema y cols $^{19}$, en 879 pacientes evaluaron el impacto clínico de los falsos negativos en la biopsia intraoperatoria por congelación. El compromiso linfonodal axilar existió en $40,8 \%$ de los casos. La sensibilidad obtenida para el estudio intraoperatorio del LC fue de $56,9 \%$ y los falsos negativos de $43,1 \%$. De los falsos negativos que fueron a disección axilar, sólo en el $15 \%$ se encontraron linfonodos metastásicos adicionales. La sobrevida a 5 años de los pacientes con falsos negativos en la biopsia por congelación fue de $90 \%$ comparado con un $94 \%$ de aquellos que no tuvieron metástasis axilares. Canavese y cols ${ }^{20}$, evaluaron la necesidad de realizar el estudio intraoperatorio por congelación del LC en 395 pacientes T1N0. Encontraron que la necesidad de una segunda intervención para la disección axilar terapéutica en el grupo sin biopsia intraoperatoria era comparable al grupo con estudio intraoperatorio $(11,6$ y $8,4 \%$, respectivamente), concluyendo que la omisión del estudio intraoperatorio en estos pacientes era segura.

Recientemente el American College of Surgeons Clinical Oncology Group (ACOSOG) en su estudio $Z 0011^{21}$, mostró que no hubo beneficio en la disección axilar de mujeres en que se les realizó tratamiento conservador, con axila negativa clínicamente y hasta dos LC positivos, lo que pone en duda la necesidad de realizar a estas pacientes la disección axilar terapéutica y más aún, la necesidad de realizar el estudio intraoperatorio del LC a pacientes con tumores pequeños y sin linfonodos axilares clínicamente detectables ${ }^{22,23}$.

\section{Conclusiones}

El rendimiento de la biopsia intraoperatoria para la detección de metástasis en el LC en nuestro centro es comparable al descrito en la literatura, siendo efectivo sólo para la pesquisa de macrometástasis (MA). Dada la baja frecuencia de metástasis en el LC en tumores de hasta $2 \mathrm{~cm}$, asociado a un número relativamente alto de MI y SM y de falsos negativos, nos parece que en estos tumores la utilización de la biopsia intraoperatoria del LC es innecesaria. Esta conducta es concordante con las recomendaciones posteriores a los resultados del American College of Surgeons Oncology Group (ACOSOG) Z0011.

\section{Referencias}

1. Krag D, Weaver D, Ashikaga T, Moffat F, Klimberg VS, Shriver C, et al. The sentinel node in breast cancer: a multicenter validation study. $\mathrm{N}$ Engl J Med. 1998;339:941-6.

2. Schwartz GF, Giuliano AE, Veronesi U; Consenus Conference Committe. Proceedings of the consensus conference on the role of sentinel lymph node biopsy in carcinoma of the breast, April 19-22, 2001, Philadelphia, Pennsylvania. Cancer. 2002;94:2542-51.

3. Edge SB, Niland JC, Bookman MA, Theriault RL, Ottesen R, Lepisto E, et al. Emergence of sentinel node biopsy in breast cancer as standard-of-care in academic comprehensive cancer centers. J Natl Cancer Inst. 2003;95:1514-21.

4. Veronesi U, Paganelli G, Viale G, Luini A, Zurrida S, Galimberti V, et al. A randomized comparison of sentinel-node biopsy with routine axillary dissection in breast cancer. N Engl J Med. 2003;349:546-53.

5. Schrenk P, Rieger R, Shamiyeh A, Wayand W. Morbidity following sentinel lymph node biopsy versus axillary lymph node dissection for patients with breast carcinoma. Cancer 2000;88:608-14.

6. Burak WE, Hollenbeck ST, Zervos EE, Hock KL, Kemp LC, Young DC. Sentinel lymph node biopsy results in less postoperative morbidity compared with axillary lymph node dissection for breast cancer. Am J Surg. 2002;183:23-7.

7. Swenson KK, Nissen MJ, Ceronsky C, Swenson L, Lee MW, Tuttle TM. Comparison of side effects between sentinel lymph node and axillary lymph node dissection for breast cancer. Ann Surg Oncol. 2002;9:745-53.

8. Golshan M, Martin WJ, Dowlatshahi K. Sentinel lymph node biopsy lowers the rate of lymphedema when compared with standard axillary lymph node dissection. Am Surg. 2003;69:209-12.

9. Lucci A, McCall LM, Beitsch PD, Whitworth PW, Reintgen DS, Blumencranz PW, et al. Surgical complications associated with sentinel lymph node dissection 
(SLND) plus axillary lymph node dissection compared with SLND alone in the American College of Surgeons Oncology Group Trial Z0011. J Clin Oncol. 2007;25:3657-63.

10. Treseler PA, Tauchi PS. Pathologic analysis of the sentinel lymph node. Surg Clin North Am. 2000;80:1695719.

11. Pargaonkar AS, Beissner RS, Snyder S, Speights VO Jr. Evaluation of immunohistochemistry and multiple-level sectioning in sentinel lymph nodes from patients with breast cancer. Arch Pathol Lab Med. 2003;127:701-5.

12. Lyman GH, Giuliano AE, Somerfield MR, Benson AB 3rd, Bodurka DC, Burstein HJ, et al. American Society of Clinical Oncology guideline recommendations for sentinel lymph node biopsy in early-stage breast cancer. J Clin Oncol. 2005;23:7703-20.

13. Liu LC, Lang JE, Lu Y, Roe D, Hwang SE, Ewing CA, et al. Intraoperative Frozen Section Analysis of Sentinel Lymph Nodes in Breast Cancer Patients. A MetaAnalysis and Single-Institution Experience. Cancer 2011;117:250-8.

14. Tew K, Irwig L, Matthews A, Crowe P, Macaskill P. Metaanalysis of sentinel node imprint cytology in breast cancer. Br J Surg. 2005;92:1068-80.

15. Camus M, León A, Schwartz E, Goñi I, Dominguez F, Oddo D, et al. Linfonodo centinela en cáncer de mama: efecto de la mayor experiencia y del uso de la técnica mixta en la mejoría de los resultados. Rev Chil Cancerología y Hematología 2004;14:125-32.

16. Cipolla C, Cabibi D, Fricano S, Vieni S, Gentile I, Latteri MA. The value of intraoperative frozen section examination of sentinel lymph nodes in surgical management of breast carcinoma. Langenbecks Arch Surg. 2010;395:685-91.

17. Weiser MR, Montgomery LL, Susnik B, Tan LK, Borgen PI, Cody HS. Is routine intraoperative frozen- section examination of sentinel lymph nodes in breast cancer worthwhile? Ann Surg Oncol. 2000;7:651-5.

18. Chan SW, LaVigne C, Port E, Fey JV, Brogi E, Borgen PJ, et al. Does the benefit of sentinel froze section vary between patients with invasive duct, invasive lobular, and favorable histologic subtypes of breast cancer? Ann Surg. 2008;247:143-9.

19. Geertsema D, Gobardhan PD, Madsen EV, Albregts M, Van Gorp J, de Hooge P, et al. Discordance of intraoperative frozen section analysis with definitive histology of sentinel lymph nodes in breast cancer surgery: complementary axillary lymph node dissection is irrelevant for subsequent systemic therapy. Ann Surg Oncol. 2010;17:2690-5.

20. Canavese G, Bruzzi P, Catturich A, Vecchio C, Tomei D, Carli F, et al. Intra-operative evaluation of the sentinel lymph node for T1-N0 breast-cancer patients: always or never? A risk/benefit and cost/benefit analysis. Eur J Surg Oncol. 2010;36:737-44.

21. Giuliano AE, Hunt KK, Ballman KV, Beitsch PD, Whitworth PW, Blumencranz PW, et al. Axillary dissection vs no axillary dissection in women with invasive breast cancer and sentinel node metastasis: a randomized clinical trial. JAMA 2011;305:569-75.

22. Caudle AS, Hunt KK, Kuerer HM, Meric-Bernstam F, Lucci A, Bedrosian I, et al. Multidisciplinary considerations in the implementation of the findings from the American college of Surgeons Oncology Group (ACOSOG) Z0011 study: A practice-changing trial. Ann Surg Oncol. 2011;18:2407-12.

23. Massimino KP, Hessman CJ, Ellis MC, Naik AM, Vetto JT. Impact of American College of surgeons Oncology Group Z0011 and National Surgical Adjuvant Breast and Bowel Project B-32 trial results on surgeon practice in the Pacific Northwest. Am J Surg. 2012;203:61822. 